\title{
Phytodiversity and Spatial Development of Urban Flora in Lokossa, Benin
}

\author{
Erick Senademi Sogbossi ${ }^{1,2,}$, Soufouyane Zakari ${ }^{2}$, Julien Gaudence Djego ${ }^{1}$ \\ ${ }^{1}$ Faculty of Agronomic Sciences, University of Abomey-Calavi, Abomey-Calavi, Benin \\ ${ }^{2}$ Institute of Geography and Land Planning, University of Abomey-Calavi, Abomey-Calavi, Benin \\ Email address: \\ ericksogbossi@gmail.com (E. S. Sogbossi), soufouyanez2@gmail.com (S. Zakari),gdjego2@gmail.com (J. G. Djego) \\ ${ }^{*}$ Corresponding author
}

\section{To cite this article:}

Erick Senademi Sogbossi, Soufouyane Zakari, Julien Gaudence Djego. Phytodiversity and Spatial Development of Urban Flora in Lokossa, Benin. International Journal of Natural Resource Ecology and Management. Vol. 5, No. 4, 2020, pp. 145-159.

doi: 10.11648/j.ijnrem.20200504.12

Received: October 12, 2020; Accepted: October 26, 2020; Published: November 11, 2020

\begin{abstract}
The need for sustainable and green cities highlights the importance to improve plant species diversity in urban areas. We assessed the phytodiversity of three management units (strata) to enhance plant species conservation planning in Lokossa City. A forest inventory was conducted in each stratum based on one-hectare sample plots. Fifty plots were distributed following a stratified random sampling approach. We found that 53 plants species belonging to 46 genera and 22 families were established in the strata. However, the flora of the city was mixed with $50.94 \%$ of native plant species. The dominant families were Leguminosae, Arecaceae, Malvaceae, Moraceae, Combretaceae, Lamiaceae and Myrtaceae. The ten most important plant species accounted for $69.31 \%$ of the total abundance. Khaya senegalensis (Desv.) A. Juss. and Mangifera indica L. were dominant plant species. Ubiquitous species accounted for $54.72 \%$ of species pool and $82.92 \%$ of all individuals. The flora of residential zone was more diversified than those in road buffer and institutional zones. Which contributed to $60.27 \%$ of all individuals. The road buffer and institutional zones flora were quite similar. Therefore, we suggested that the planting and monitoring projects should be detailed and budgeted with reference to plausible scientific knowledge in the city development plan. The creation of participatory botanical gardens at the city neighborhoods scale. The integration of the flora of residential zone in species conservation projects in order to increase global diversity and storage of biomass in the city.
\end{abstract}

Keywords: Stratified Random Sampling, Phytodiversity, Species Differentiation, Similarity, Urban Flora, Sustainable Development

\section{Introduction}

Urban forests are essential to the functioning and stability of the urban ecosystem $[1,2]$. They contribute to the greening of cities and provide important aesthetic, social, economic and environmental services and values, most of which are not mutually exclusive $[3,4]$. Urban forests also provide habitats for many organisms (shelters, hiding places, nests, etc.), which significantly increases urban species diversity [5, 6]. Plant species diversity is a key element in the strategic management of urban forests [7, 8]. High plant species diversity enhances pest control, protection against disturbances such as climate change, the spread of insects, diseases and pest epidemics
[9-11]. Moreover, diversity influences the microclimate and thermal comfort of urban environments [12-14]. The conservation and efficient management of urban flora are imperatives to improve the quality of the environment and the well-being of cities' inhabitants [5, 15].

Yet, despite the universally known utilities of urban flora, urban forests are still subject to abiotic constraints and disturbances [16]. Furthermore, the urban population growth at $4 \%$ rate per decade by 2050 [17], doesn't help to improve the situation. These factors associated with the complex filters of urbanization [18-21], can lead to a homogenization of the flora through the expansion of exotic species and the decline of native species $[5,22]$. This process affects the ecological integrity of urban ecosystems $[9,23,24]$. The lack or failure of 
urban forest management policies in developing countries [25] affects the structure, diversity and composition of urban forests $[21,26]$, whose plant heritage remains poorly known $[27,28]$. This situation is common to many developing countries where there is still a lot to learn and achieve in terms of sustaining and improving existing species diversity and building green cities.

In Benin, a country located on the West coast of Africa, there are no local regulations that protect urban forests or conserve urban flora. Yet, over the past few decades, there is increasing awareness campaigns among the populations to demonstrate that plant species play an important role in the urban ecosystems and need to be protected. In Lokossa a typical city of Benin, the development plan is very little committed to natural resource conservation [29]. It contains only one literary paragraph which gives no information about the physiognomic, structural and compositional characteristics, functions, services and benefits of urban forests. No concrete action for protecting these forests is mentioned and the conservation of flora is absent. This highlights the lack of knowledge of the species pool of city. Although the population recognize the services provided by plant species, they don't actively engage in their conservation. In addition, when there are reforestation initiatives like for the world trees day on each June $1^{\text {st }}$, some species are planted in city ignoring precisely their capacity to adapt to changes in the environment and their ability to improve the urban climate. Most of the studies conducted in Benin have focused on streets forests [29-31]. They have also paid very little attention to assessing species differentiation between urban strata. The heterogeneity of urban environments requires significant scientific data on all plant communities for a sustainable management [32-34]. Assessing the flora of these urban forests provides indicators to support forest management and improve ecological services [35]. The main goal of this study was to assess the phytodiversity of three management units of the municipality of Lokossa to ensure better plant species conservation planning.

\section{Study Area}

The urban area of Lokossa is located at latitude $6^{\circ} 36^{\prime} 56^{\prime \prime}-6^{\circ} 40^{\prime} 28^{\prime \prime} \mathrm{N}$ and longitude $1^{\circ} 41^{\prime} 55^{\prime \prime}-1^{\circ} 44^{\prime} 18^{\prime \prime} \mathrm{E}$ in southwest Benin. It is a medium-size city which has experienced a rapid growth of its population in last decade. The city is situated in the Guineo-Congolese zone. The rainfall regime is bimodal with sub-tropical climate. The mean annual rainfall and temperature are $1100 \mathrm{~mm}$ and $27.91{ }^{\circ} \mathrm{C}$, respectively. Soils are mainly ferralitic on loose clayey sediment. These climatic and pedagogical conditions are favorable to the establishment of diversified flora in the city. The urban area of Lokossa covers $15 \mathrm{~km}^{2}$ currently, with a population density of 874 persons per $\mathrm{km}^{2}$ and an average annual growth rate of $2.23 \%$ [36]. This rapid population growth rate is a determinant factor of species community's disturbance. Therefore, it becomes urgent to know the nature of the flora and work on its integration in the city development plan in the context of global changes.

\section{Material and Methods}

\subsection{Forest Inventory}

The stratified random sampling approach [37] was used for the forest inventory. This approach was adopted for a relatively easy assessment of urban forest monitoring [37]. This is a part of sampling approach adopted in the study of carbon stock modeling in progress. Moreover, the city was post-stratified into three management units (urban strata) based on the flora management practices and the plant-canopy cover per hectare with reference to land use and location [38]. Plant-canopy cover recognition and delineation were made by aerial photographs at 1:2500 scale. This aerial-survey associated with management practices allowed us to group the urban forests into three strata namely institutional zone (High canopy cover), road buffer zone (medium canopy cover) and residential zone (low canopy cover). According to management practices, the flora of institutional zone was better maintained and protected by site managers. The flora of road buffer zone was less maintained, but had formal legal status. Which, was considerably reduced the human exploitation of them. As for the residential zone flora, it was governed by the populations' attitude and cultural norms. It was freer of access, and under high anthropogenic disturbances. With regards to the spatial structure of the strata, the institutional zone (IZ) was reference to all public, private and religious administrative compounds. The road buffer zone (RBZ) was the area cover by a delineation of buffer of $50 \mathrm{~m}$ around road networks. The residential zone (RZ) was composed of the space between the two others strata with exception to stream, water body, swamp and bare soils.

The functional types of flora considered in this forest inventory were trees, shrubs and palms. We used the square sample plots of one-hectare size for assessing plant species composition and diversity in each stratum. Fifty plots were prospected in the three strata, i.e. an area of 50 ha. A sample plot belonged to one or another stratum when it is at least for $50 \%$ included in the stratum limits [39]. The free version of applications Mobil Topographer 9.3.2 and QField 1.5.3 were used to locate and move in the sample plots area, respectively. In each sample plot, all plant species with Diameter at Breast Height $(\mathrm{DBH}) \geq 5 \mathrm{~cm}$ were identified at species level, counted by species and their diameters were measured using calipers or a diameter tape. For plants with multiple stems below $1.3 \mathrm{~m}$; the diameter of each stem was measured and their combined mean calculated as the quadratic mean of the individual stem [21, 32]. The species in the plots were identified with the help of plants experts in the National Herbarium of Abomey-Calavi University (UAC). The chorological distribution of plant species was used to identify the origin of each plant recorded.

\subsection{Data Analysis}

The floristic composition of the strata was examined using: frequencies of botanical families, genera and taxonomic classes of plant species; origin of plant species (exotic or native); 
commonality and uniqueness of plant species. Descriptive statistics were used to determine the proportion of taxa per taxonomic classes, families, genera and origin in each stratum [32]. To get the level of floristic difference between strata based on the number of plant species and species importance value per family, we used the Spearman rank correlation coefficient. Venn diagram was used to visualize the number of plant species between commonality groups. Fisher's exact test [40] and Cramer's V statistic were used to assess the degree of association (at $5 \%$ threshold) between strata.

To assess the shifting in the species composition of strata, the Importance Value Index (IVI) of each species was computed [41]. For a species $\alpha$, the IVI was calculated using Equation (1).

$$
I V I_{\alpha}=\left(R A_{\alpha}+R D_{\alpha}+R F_{\alpha}\right) \times 100
$$

where $\mathrm{RA}_{\alpha}$ is the relative abundance of specie $\alpha$ as a number of individuals of the specie /total number of individuals of all species; $\mathrm{RD}_{\alpha}$ is the relative dominance of specie $\alpha$ as a basal area at breast height of the specie/total basal area of all species; $\mathrm{RF}_{\alpha}$ is the relative frequency of specie $\alpha$ as a frequency of the specie/sum of all frequencies.

The values of IVI may range from 0 to 300 . It gives an overall estimate of the level of importance of a plant species in the community [42]. The coefficient of correlation of the IVI values between strata was computed. Cluster analysis was performed using R4.0.1 software in order to analyze the trend of species importance value among strata. The four established groups were projected in the system axes of the Principal Component Analysis (PCA) on the IVI values in the three strata.

In this study, plant species diversity was assessed using $\alpha$-diversity, $\beta$-diversity and evenness [43]. The alpha diversity was analyzed through the plant species richness (S), Shannon-Weaver diversity index $\left(H^{\prime}\right)$, Pileou's evenness index $\left(J^{\prime}\right)$ and Simpson's diversity index $(I D S)$ [44].

The species richness $(S)$ was the number of plant species recorded in each stratum.

The Shannon-Weaver diversity index $\left(H^{\prime}\right)$ was calculated for each stratum as follows (Equation (2)).

$$
H^{\prime}=\sum_{i=1}^{s} p_{i} \ln \left(p_{i}\right)
$$

where $p_{i}$ is the count proportion of the $i$ th species in the stratum under consideration and $\ln \left(p_{i}\right)$ represents the natural logarithm of this proportion.

The Shannon-Weaver diversity index varying from 0 (communities with only a single taxon) to high values for communities with many taxa, each with similar abundance [43].

The Shannon diversity index was compared between strata based on Equations from (3) to (5) according to [45]:

The variance of $H^{\prime}$ ( $v a r H^{\prime}$ ) was calculated as follows:

$$
\operatorname{var} H^{\prime}=\frac{\sum_{i=1}^{s} p_{i}\left(\ln \left(p_{i}\right)\right)^{2}-\left(\sum_{i=1}^{s} p_{i} \ln \left(p_{i}\right)\right)^{2}}{n}-\frac{S-1}{2 n^{2}}
$$

where $n$ is a number of all individuals in the stratum.

The t-statistic for testing the significant difference between two strata was:

$$
t=\left(H_{1}^{\prime}-H_{2}^{\prime}\right) / \sqrt{\operatorname{var} H_{1}^{\prime}+\operatorname{var} H_{2}^{\prime}}
$$

where $H_{j}^{\prime}$ represents the Shannon's diversity index for any stratum $j$.

The degree of freedom $(d d l)$ of the test was equal to:

$$
d d l=\frac{\left(\operatorname{var} H_{1}^{\prime}+\operatorname{var} H_{2}^{\prime}\right)^{2}}{\left(\operatorname{var} H_{1}^{\prime}\right)^{2} / n_{1}+\left(\operatorname{var} H_{2}^{\prime}\right)^{2} / n_{2}}
$$

where $n_{1}$ is the total of individuals in stratum 1 , and $n_{2}$ is the total number of individuals in stratum 2 .

The Pielou evenness index $\left(J^{\prime}\right)$ was obtained for each stratum based on Equation (6).

$$
J^{\prime}=H^{\prime} / H_{\max }^{\prime}=H^{\prime} / \ln (S)
$$

where $H_{\max }^{\prime}=\ln (S)$ represents the Shannon's maximum entropy.

Pielou's evenness index varying from 0 and 1 . It is 0 when there is a phenomenon of dominance and 1 when the distribution of individuals among species is homogenous.

The Simpson's diversity index (SDI) was determined for each stratum using Equation (7).

$$
S D I=1-\sum_{i-1}^{s} \frac{n_{i}\left(n_{i}-1\right)}{n(n-1)}
$$

where $n_{i}$ is the number of individuals of $i$ th plant species in the stratum under consideration and $n$ represents the total number of individuals in the stratum.

Simpson's diversity index ranging from 0 (communities with low diversity) to 1 for communities with higher diversity level [43].

The compositional similarity between strata was performed through beta diversity analysis. The beta diversity was estimated using the reformulated Sørenson and Jaccard indices [46]. These indices are based on a probabilistic approach which combines the incidence-based indices with relative abundance data, thus minimizing bias through unequal weighting of rare and common species. The computation of these indices and their estimators were described in [46, 47]. The values of these two adjusted indices range from 0 to 1 , with 0 indicating absolute dissimilarity and 1 indicating absolute similarity [47]. The High values of these indices show low beta diversity (high similarity) and low values show high beta diversity (high dissimilarity) $[21,26]$. Confidence intervals $(95 \% \mathrm{CI})$ of the similarity indices were estimated by Bootstrap after 1000 replications.

The R4.0.1 software [48] was used for the statistical analysis and the computation of all diversity indices. 


\section{Results}

\subsection{Taxonomic Diversity of Plant Species}

Overall 896 plants species $(79.02 \%$ of trees, $8.15 \%$ of shrubs and $12.83 \%$ of palms) were sampled across the strata. These plant species were grouped into 53 species belonging to 46 genera and 22 families. They were distributed among two major taxonomic classes with $92.45 \%$ of Dicotyledonous and $7.55 \%$ of Monocotyledonous. The most represented families in three strata were Leguminosae (15.09\% of plant species), Arecaceae (7.55\% of plant species), Malvaceae (7.55\% of plant species), Moraceae $(7.55 \%$ of plant species), Combretaceae (5.66\% of plant species), Lamiaceae (5.66\% of plant species) and Myrtaceae (5.66\% of plant species).

The residential zone had the highest taxonomic diversity. The best represented families in this stratum were Leguminosae (13.95\% of plant species), Arecaceae (9.30\% of plant species),

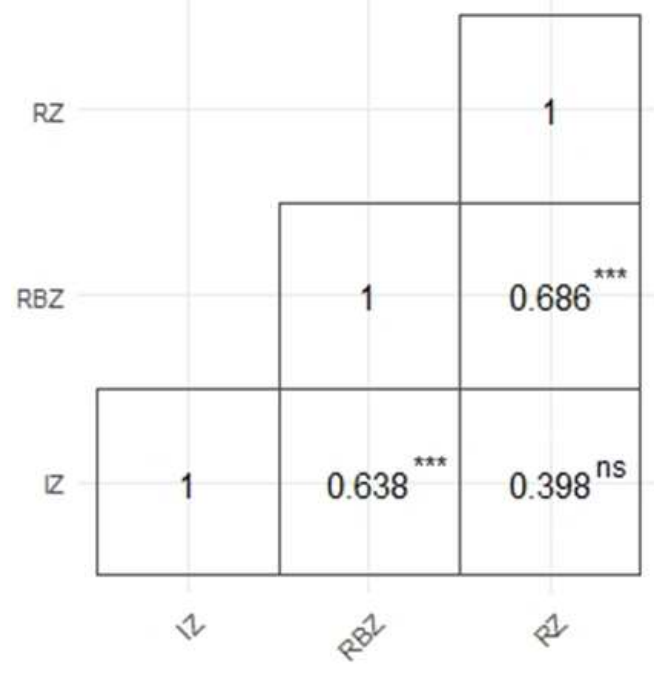

(a) Species count per family
Moraceae (9.30\% of plant species), Combretaceae $(6.98 \%$ of plant species) and Lamiaceae (6.98\% of plant species). In the road buffer zone, Leguminosae (15.38\% of plant species) and Lamiaceae (11.54\% of plant species) were predominant. Regarding the institutional zone, $11.54 \%$ of the plant species were equivalently encountered in the three families Arecaceae, Combretaceae and Malvaceae.

The flora of the road buffer zone was a highly correlated (p-value $=0.000)$ taxonomic diversity with those of both institutional and residential zones (Figure 1a). On the other hand, no significant correlation was founded between the institutional and residential zones. According to the species importance value index (IVI), there was a very strong correlation $(p$-value $=0.000)$ between the road buffer and residential zones. This correlation was less strong between the road buffer and institutional zones. The Institutional and residential zones exhibited relatively low correlation (Figure 1b).

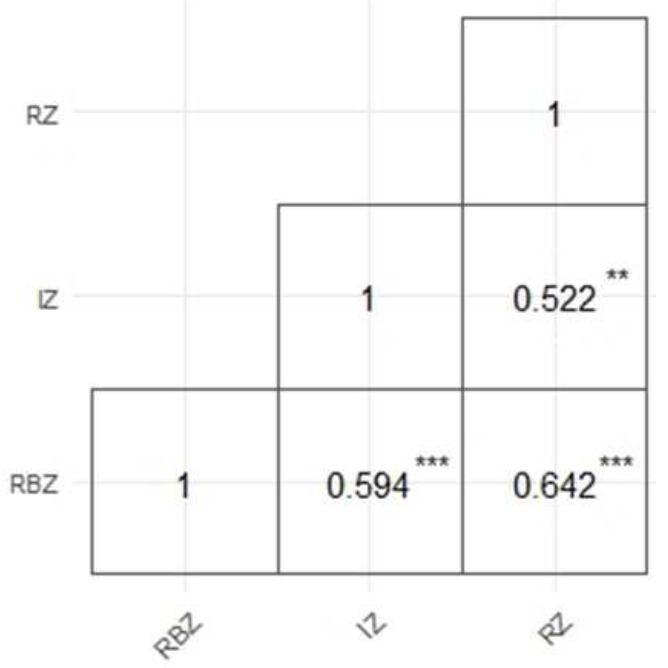

(b) IVI of species per family

Figure 1. Spearman rank correlation of plant species count (a) and plant species importance value index (b) per family between urban strata (RZ-Residential zone, IZ-Institutional zone, RBZ-Road buffer zone, ***: Significance level $<0.001$, **Significance level $<0.05$, and ns: Not significant at 0.05).

\subsection{Plant Species Importance Value}

As far as the species composition of the urban strata was concerned, the species pool had 20 plant species with value of IVI $\geq 10 \%$ (Table 1 ). Fourteen of these species had a value of IVI ranging between 13 to $58 \%$ (Table 1 ). The coefficient of correlation based on the IVI values between the three strata varied from 0.16 to 0.51 (Table 2). The residential and institutional zones exhibited none significant correlation.

Moreover, four groups of plant species were established from the cluster analysis performed on the IVI values and $88.84 \%$ of the information on the IVI values of the plant species in the three strata was saved. The projection of the four groups in the axe systems obtained from the principal component analysis performed on the IVI values showed that the first axis discriminated the groups of plant species with high IVI values from those of low values of IVI (Figure 2). Therefore, the cluster 1 was constituted of plant species of low IVI values such as Jatropha multifida L., Gardenia ternifolia
Schumach. \& Thonn., Commiphora Africana (A. Rich.) Engl., Borassus aethiopum Mart., Tephrosia vogelii Hook. $f$. (Table 1). The clusters 3 and 4 were constituted of plant species with high IVI values (Khaya senegalensis (Desv.) A. Juss., Azadirachta indica A. Juss. and Terminalia catappa L. for cluster 3, and Mangifera indica L., Cocos nucifera L., Citrus sinensis (L.) Osbeck and Ficus umbellata Vahl for cluster 4). The second axis distinguished groups of plant species according to their value of IVI in each stratum, respective of management practices and plant-canopy cover. Therefore, the cluster 3 was composed of plant species with high plant-canopy cover in institutional zone. The cluster 4 was constituted of plant species with low plant-canopy in residential zone. An intermediate trend was observed for the cluster 2 which was composed of plant species with moderate values of IVI and plant-canopy cover in road buffer zone. Among them were Gliricidia sepium (Jacq.) Walp., Moringa oleifera Lam., Tectona grandis L. f., Gmelina arborea Roxb. and Ceiba pentandra (L.) Gaertn. 
Table 1. Importance value index, origin and family of plant species in the urban strata.

\begin{tabular}{|c|c|c|c|c|c|c|c|}
\hline Strata & Origin & Plant species & Family & $\mathbf{R A}_{\alpha}$ & $\mathbf{R D}_{\alpha}$ & $\mathbf{R F}_{\boldsymbol{\alpha}}$ & $\mathbf{I V I}_{\alpha}$ \\
\hline RBZ & Exotic & & & 71.12 & 76.45 & 71.43 & 219.00 \\
\hline RBZ & Exotic & Cocos nucifera $L$. & Arecaceae & 15.09 & 7.36 & 12.99 & 35.44 \\
\hline RBZ & Exotic & Ceiba pentandra (L.) Gaertn. (b) & Malvaceae & 0.86 & 29.78 & 1.30 & 31.94 \\
\hline RBZ & Exotic & Terminalia catappa $L$ & Combretaceae & 8.62 & 7.71 & 6.49 & 22.83 \\
\hline RBZ & Exotic & Azadirachta indica A. Juss. & Meliaceae & 9.48 & 6.53 & 6.49 & 22.51 \\
\hline RBZ & Exotic & Mangifera indica $L$. & Anacardiaceae & 6.90 & 5.36 & 9.09 & 21.34 \\
\hline RBZ & Exotic & Gmelina arborea Roxb. & Lamiaceae & 8.19 & 5.84 & 5.19 & 19.22 \\
\hline RBZ & Exotic & Citrus sinensis (L.) Osbeck & Rutaceae & 5.60 & 1.28 & 9.09 & 15.98 \\
\hline RBZ & Exotic & Tectona grandis L. $f$. & Lamiaceae & 6.03 & 4.82 & 3.90 & 14.75 \\
\hline RBZ & Exotic & Terminalia mantaly H. Perrier & Combretaceae & 3.88 & 5.36 & 2.60 & 11.84 \\
\hline RBZ & Exotic & Moringa oleifera Lam. & Moringaceae & 3.88 & 0.38 & 6.49 & 10.75 \\
\hline RBZ & Exotic & Persea americana Mill. & Lauraceae & 1.29 & 0.71 & 3.90 & 5.90 \\
\hline RBZ & Exotic & Delonix regia (Boj. ex Hook.) Raf. & Leguminosae & 0.43 & 1.26 & 1.30 & 2.99 \\
\hline RBZ & Exotic & Psidium guajava $L$ & Myrtaceae & 0.43 & 0.03 & 1.30 & 1.76 \\
\hline RBZ & Exotic & Citrus limon (L.) Osbeck & Rutaceae & 0.43 & 0.02 & 1.30 & 1.75 \\
\hline RBZ & Native & & & 28.88 & 23.55 & 28.57 & 81.00 \\
\hline RBZ & Native & Khaya senegalensis (Desv.) A. Juss. & Meliaceae & 9.05 & 7.42 & 7.79 & 24.27 \\
\hline RBZ & Native & Ficus umbellata Vahl & Moraceae & 7.33 & 5.92 & 3.90 & 17.14 \\
\hline RBZ & Native & Elaeis guineensis Jacq. & Arecaceae & 5.17 & 5.92 & 5.19 & 16.29 \\
\hline RBZ & Native & Newbouldia laevis (P. Beauv.) Seem. & Bignoniaceae & 2.16 & 0.41 & 1.30 & 3.86 \\
\hline RBZ & Native & Ficus ingens (Miq.) Miq. & Moraceae & 2.16 & 0.06 & 1.30 & 3.51 \\
\hline RBZ & Native & Vitex doniana Sweet & Lamiaceae & 0.43 & 1.57 & 1.30 & 3.30 \\
\hline RBZ & Native & Vachellia amythethophylla (Steud. ex A. Rich.) Kyal. \& Boatwr. & Leguminosae & 0.43 & 1.13 & 1.30 & 2.86 \\
\hline RBZ & Native & Morinda lucida Benth. & Rubiaceae & 0.43 & 0.46 & 1.30 & 2.19 \\
\hline RBZ & Native & Blighia sapida K. D. Koenig & Sapindaceae & 0.43 & 0.34 & 1.30 & 2.06 \\
\hline RBZ & Native & Senna siamea (Lam.) H. S. Irwin \& Barneby & Leguminosae & 0.43 & 0.26 & 1.30 & 1.99 \\
\hline RBZ & Native & Cola millenii K. Schum. & Malvaceae & 0.43 & 0.04 & 1.30 & 1.77 \\
\hline RBZ & Native & Erythrina senegalensis DC. & Leguminosae & 0.43 & 0.04 & 1.30 & 1.77 \\
\hline IZ & Exotic & & & 64.52 & 57.42 & 57.78 & 179.72 \\
\hline IZ & Exotic & Azadirachta indica A. Juss. & Meliaceae & 8.06 & 17.28 & 6.67 & 32.02 \\
\hline $\mathrm{IZ}$ & Exotic & Mangifera indica $L$. & Anacardiaceae & 11.29 & 11.84 & 8.89 & 32.01 \\
\hline IZ & Exotic & Terminalia catappa $L$. & Combretaceae & 9.68 & 13.45 & 4.44 & 27.58 \\
\hline IZ & Exotic & Cocos nucifera L. & Arecaceae & 8.87 & 5.33 & 8.89 & 23.09 \\
\hline IZ & Exotic & Citrus sinensis (L.) Osbeck & Rutaceae & 11.29 & 2.22 & 8.89 & 22.4 \\
\hline IZ & Exotic & Polyalthia longifolia (Sonn.) Thwaites ${ }^{(c)}$ & Annonaceae & 5.65 & 0.95 & 4.44 & 11.04 \\
\hline IZ & Exotic & Terminalia superba Engl. \& Diels & Combretaceae & 2.42 & 1.04 & 2.22 & 5.68 \\
\hline IZ & Exotic & Terminalia mantaly H. Perrier & Combretaceae & 0.81 & 2.52 & 2.22 & 5.55 \\
\hline IZ & Exotic & Eucalyptus torrelliana (F. Muell.) K. D. Hill \& L. A. S. Johnson ${ }^{(c)}$ & Myrtaceae & 1.61 & 1.62 & 2.22 & 5.45 \\
\hline IZ & Exotic & Picea abies (L.) H. Karst. ${ }^{(c)}$ & Pinaceae & 1.61 & 0.67 & 2.22 & 4.5 \\
\hline IZ & Exotic & Delonix regia (Boj. ex Hook.) Raf. & Leguminosae & 1.61 & 0.12 & 2.22 & 3.96 \\
\hline IZ & Exotic & Persea americana Mill. & Lauraceae & 0.81 & 0.2 & 2.22 & 3.23 \\
\hline IZ & Exotic & Citrus limon (L.) Osbeck & Rutaceae & 0.81 & 0.18 & 2.22 & 3.20 \\
\hline IZ & Native & & & 35.48 & 42.58 & 42.22 & 120.28 \\
\hline IZ & Native & Khaya senegalensis (Desv.) A. Juss. & Meliaceae & 17.74 & 28.41 & 11.11 & 57.26 \\
\hline IZ & Native & Triplochiton scleroxylon K. Schum. ${ }^{(c)}$ & Malvaceae & 5.65 & 1.80 & 4.44 & 11.89 \\
\hline IZ & Native & Elaeis guineensis Jacq. & Arecaceae & 1.61 & 1.15 & 4.44 & 7.21 \\
\hline IZ & Native & Vachellia amythethophylla (Steud. ex A. Rich.) Kyal. \& Boatwr. & Leguminosae & 0.81 & 3.52 & 2.22 & 6.55 \\
\hline $\mathrm{IZ}$ & Native & Ficus umbellata Vahl & Moraceae & 0.81 & 3.26 & 2.22 & 6.29 \\
\hline IZ & Native & Borassus aethiopum Mart. & Arecaceae & 0.81 & 2.3 & 2.22 & 5.33 \\
\hline IZ & Native & Croton gratissimus Burch. & Euphorbiaceae & 2.42 & 0.14 & 2.22 & 4.79 \\
\hline $\mathrm{IZ}$ & Native & Rhodognaphalon brevicuspe (Sprague) Roberty ${ }^{(c)}$ & Malvaceae & 1.61 & 0.68 & 2.22 & 4.51 \\
\hline IZ & Native & Ficus ingens (Miq.) Miq. & Moraceae & 0.81 & 0.69 & 2.22 & 3.72 \\
\hline IZ & Native & Spondias mombin L. & Anacardiaceae & 0.81 & 0.21 & 2.22 & 3.24 \\
\hline $\mathrm{IZ}$ & Native & Cola millenii K. Schum. & Malvaceae & 0.81 & 0.19 & 2.22 & 3.22 \\
\hline IZ & Native & Holarrhena floribunda (G. Don) T. Durand \& Schinz ${ }^{(c)}$ & Apocynaceae & 0.81 & 0.15 & 2.22 & 3.18 \\
\hline $\mathrm{IZ}$ & Native & Vitex doniana Sweet & Lamiaceae & 0.81 & 0.08 & 2.22 & 3.11 \\
\hline $\mathrm{RZ}$ & Exotic & & & 71.48 & 53.19 & 62.23 & 186.91 \\
\hline $\mathrm{RZ}$ & Exotic & Mangifera indica $L$. & Anacardiaceae & 11.11 & 13.63 & 7.98 & 32.72 \\
\hline $\mathrm{RZ}$ & Exotic & Cocos nucifera $L$ & Arecaceae & 7.96 & 12.83 & 7.98 & 28.77 \\
\hline $\mathrm{RZ}$ & Exotic & Gliricidia sepium (Jacq.) Walp. ${ }^{(a)}$ & Leguminosae & 14.26 & 2.95 & 5.32 & 22.53 \\
\hline $\mathrm{RZ}$ & Exotic & Citrus sinensis (L.) Osbeck & Rutaceae & 9.26 & 2.52 & 6.91 & 18.7 \\
\hline RZ & Exotic & Moringa oleifera Lam. & Moringaceae & 7.22 & 1.65 & 5.85 & 14.72 \\
\hline $\mathrm{RZ}$ & Exotic & Azadirachta indica A. Juss. & Meliaceae & 4.26 & 4.67 & 4.79 & 13.72 \\
\hline $\mathrm{RZ}$ & Exotic & Tectona grandis L. $f$. & Lamiaceae & 2.41 & 2.37 & 2.13 & 6.91 \\
\hline $\mathrm{RZ}$ & Exotic & Acacia auriculiformis Cunn. ex Benth. ${ }^{(a)}$ & Leguminosae & 2.41 & 2.78 & 1.6 & 6.78 \\
\hline
\end{tabular}




\begin{tabular}{|c|c|c|c|c|c|c|c|}
\hline Strata & Origin & Plant species & Family & $\mathbf{R A _ { \alpha }}$ & $\mathbf{R D}_{\alpha}$ & $\mathbf{R F}_{a}$ & IVI $_{a}$ \\
\hline $\mathrm{RZ}$ & Exotic & Terminalia catappa $L$. & Combretaceae & 0.74 & 2.84 & 2.13 & 5.70 \\
\hline RZ & Exotic & Annona muricata $L .^{(a)}$ & Annonaceae & 1.85 & 0.53 & 3.19 & 5.57 \\
\hline RZ & Exotic & Citrus limon (L.) Osbeck & Rutaceae & 2.04 & 0.34 & 3.19 & 5.57 \\
\hline $\mathrm{RZ}$ & Exotic & Terminalia superba Engl. \& Diels & Combretaceae & 1.48 & 1.24 & 2.13 & 4.85 \\
\hline RZ & Exotic & Psidium guajava $L$. & Myrtaceae & 1.67 & 0.35 & 2.13 & 4.14 \\
\hline $\mathrm{RZ}$ & Exotic & Terminalia mantaly H. Perrier & Combretaceae & 0.37 & 2.18 & 1.06 & 3.62 \\
\hline $\mathrm{RZ}$ & Exotic & Gmelina arborea Roxb. & Lamiaceae & 1.30 & 0.26 & 1.60 & 3.15 \\
\hline $\mathrm{RZ}$ & Exotic & Persea americana Mill. & Lauraceae & 0.74 & 0.63 & 1.60 & 2.97 \\
\hline $\mathrm{RZ}$ & Exotic & Eucalyptus camaldulensis Dehnh. ${ }^{(a)}$ & Myrtaceae & 1.30 & 0.90 & 0.53 & 2.73 \\
\hline $\mathrm{RZ}$ & Exotic & Adonidia merrillii (Becc.) Becc. ${ }^{(a)}$ & Arecaceae & 0.56 & 0.02 & 0.53 & 1.11 \\
\hline $\mathrm{RZ}$ & Exotic & Artocarpus altilis (Parkinson ex F. A. Zorn) Fosberg ${ }^{(a)}$ & Moraceae & 0.19 & 0.29 & 0.53 & 1.01 \\
\hline $\mathrm{RZ}$ & Exotic & Crescentia cujete L. ${ }^{(a)}$ & Bignoniaceae & 0.19 & 0.22 & 0.53 & 0.93 \\
\hline RZ & Exotic & Jatropha multifida L. ${ }^{(a)}$ & Euphorbiaceae & 0.19 & 0.00 & 0.53 & 0.72 \\
\hline $\mathrm{RZ}$ & Native & & & 28.52 & 46.81 & 37.77 & 113.09 \\
\hline $\mathrm{RZ}$ & Native & Ficus umbellata Vahl & Moraceae & 7.59 & 22.21 & 8.51 & 38.31 \\
\hline $\mathrm{RZ}$ & Native & Ficus ingens (Miq.) Miq. & Moraceae & 6.11 & 2.73 & 4.79 & 13.63 \\
\hline $\mathrm{RZ}$ & Native & Elaeis guineensis Jacq. & Arecaceae & 1.30 & 8.70 & 2.66 & 12.66 \\
\hline $\mathrm{RZ}$ & Native & Khaya senegalensis (Desv.) A. Juss. & Meliaceae & 1.48 & 6.75 & 2.13 & 10.36 \\
\hline $\mathrm{RZ}$ & Native & Spondias mombin $L$. & Anacardiaceae & 1.67 & 0.73 & 4.26 & 6.66 \\
\hline RZ & Native & Senna siamea (Lam.) H. S. Irwin \& Barneby & Leguminosae & 2.22 & 1.47 & 2.13 & 5.82 \\
\hline RZ & Native & Newbouldia laevis (P. Beauv.) Seem. & Bignoniaceae & 1.11 & 0.42 & 2.13 & 3.66 \\
\hline $\mathrm{RZ}$ & Native & Crateva adansonii DC. ${ }^{(a)}$ & Capparaceae & 1.11 & 0.64 & 1.60 & 3.35 \\
\hline $\mathrm{RZ}$ & Native & Erythrina senegalensis $D C$. & Leguminosae & 2.22 & 0.26 & 0.53 & 3.02 \\
\hline $\mathrm{RZ}$ & Native & Croton gratissimus Burch. & Euphorbiaceae & 0.56 & 0.15 & 1.60 & 2.30 \\
\hline RZ & Native & Blighia sapida K. D. Koenig & Sapindaceae & 0.37 & 0.34 & 1.06 & 1.78 \\
\hline $\mathrm{RZ}$ & Native & Lecaniodiscus cupanioides Planch. ex Benth. ${ }^{(a)}$ & Sapindaceae & 0.19 & 0.86 & 0.53 & 1.58 \\
\hline $\mathrm{RZ}$ & Native & Vitex doniana Sweet & Lamiaceae & 0.19 & 0.82 & 0.53 & 1.53 \\
\hline $\mathrm{RZ}$ & Native & Morinda lucida Benth. & Rubiaceae & 0.37 & 0.08 & 1.06 & 1.52 \\
\hline RZ & Native & Ficus exasperata Vahl (a) & Moraceae & 0.56 & 0.12 & 0.53 & 1.21 \\
\hline $\mathrm{RZ}$ & Native & Calotropis procera (Aiton) Dryand. ${ }^{(a)}$ & Apocynaceae & 0.37 & 0.08 & 0.53 & 0.98 \\
\hline $\mathrm{RZ}$ & Native & Parkia biglobosa (Jacq.) R. Br. ex Benth ${ }^{(a)}$ & Leguminosae & 0.19 & 0.19 & 0.53 & 0.91 \\
\hline $\mathrm{RZ}$ & Native & Tephrosia vogelii Hook. $f^{(a)}$ & Leguminosae & 0.19 & 0.10 & 0.53 & 0.81 \\
\hline RZ & Native & Borassus aethiopum Mart. & Arecaceae & 0.19 & 0.08 & 0.53 & 0.80 \\
\hline $\mathrm{RZ}$ & Native & Commiphora africana (A. Rich.) Engl. ${ }^{(a)}$ & Burseraceae & 0.19 & 0.03 & 0.53 & 0.75 \\
\hline $\mathrm{RZ}$ & Native & Dracaena arborea (Willd.) Link ${ }^{(a)}$ & Asparagaceae & 0.19 & 0.03 & 0.53 & 0.75 \\
\hline RZ & Native & Gardenia ternifolia Schumach. \& Thonn. ${ }^{(a)}$ & Rubiaceae & 0.19 & 0.00 & 0.53 & 0.72 \\
\hline
\end{tabular}

Relative abundance $\left(\mathrm{RA}_{\alpha}\right)$, Relative dominance $\left(\mathrm{RD}_{\alpha}\right)$, Relative frequency $\left(\mathrm{RF}_{\alpha}\right)$ and Importance Value Index $\left(\mathrm{IVI}_{\alpha}\right)$ for each species $\alpha$. The species only present in residential zone (RZ), road buffer zone (RBZ) and institutional zone (IZ) were associated with the letters (a), (b) and (c), respectively.

Table 2. Spearman rank correlation of plant species importance value between urban strata.

\begin{tabular}{llll}
\hline Urban strata & RBZ & IZ & RZ \\
\hline $\mathrm{RBZ}$ & 1 & & \\
$\mathrm{IZ}$ & $0.40^{* * *}$ & 1 & \\
$\mathrm{RZ}$ & $0.51^{* * *}$ & $0.16^{\mathrm{ns}}$ & 1 \\
\hline
\end{tabular}

***: Significance level $<0.001$; ns: Not significant at 0.05 .

\subsection{Plant Species Differentiation}

The native species accounted for $50.94 \%$ of species pool. The analysis conducted by stratum showed that the institutional zone had almost equivalent number of exotic and native plant species, whereas the exotic/native plant species ratio was 0.95 in the residential zone and 1.17 in the road buffer zone. However, there was a very large variation in the plant species abundance. The proportion of native plant species was higher in the residential zone than the two others. Of the eight (8) most important plant species in residential zone, seven (7) were exotic. The number of the most important exotic plant species in the institutional and road buffer zones, was six (6) and five (5), respectively. The exotic plant species had a higher importance value than the native ones with a ratio of 1.49 in the institutional zone, 1.65 in the residential zone and 2.70 in road buffer zone. Furthermore, the residential zone provided refuge for native plant species with extremely low relative frequency than the institutional and road buffer zones (Table 1).

\subsection{Plant Species Commonality and Uniqueness}

The decomposition of plant species pool between strata revealed seven commonality groups (Figure 3). The plant species common to two or three strata accounted for $54.72 \%$ of species pool. Those belonging to only one stratum were $45.28 \%$ (Table 3 ). Thirteen plant species were common to the three strata (Figure 3).

The residential zone (RZ) shared 22 and 17 plant species with the road buffer zone (RBZ) and the institutional zone (IZ), respectively. The institutional zone shared 16 plant species with the road buffer zone. The Road buffer zone's plant species were almost present in the two other strata except one plant (Ceiba pentandra). 


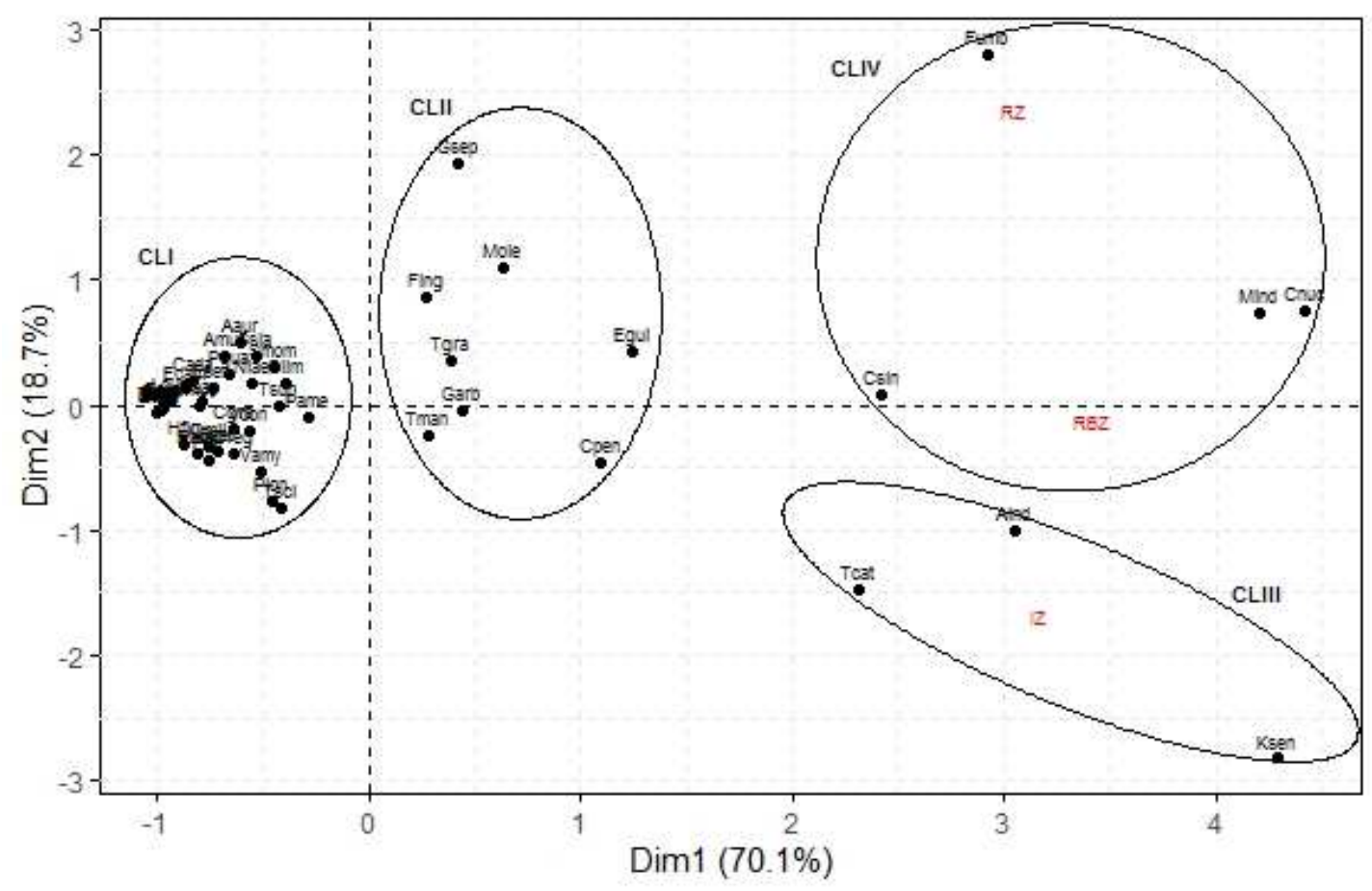

Figure 2. Projection of the four groups of plant species according to the IVI values in the system axes of PCA. CLI to CLIV: Cluster one to four. Urban strata: IZInstitutional zone, RBZ-Road buffer zone, RZ-Residential zone. Plant species: Aaur = Acacia auriculiformis, Amer = Adonidia merrillii, Amur = Annona muricata, Aalt $=$ Artocarpus altilis, Aind = Azadirachta indica, Bsap = Blighia sapida, Baet $=$ Borassus aethiopum, Cpro $=$ Calotropis procera, Cpen $=$ Ceiba pentandra, Clim = Citrus limon, Csin $=$ Citrus sinensis, Cnuc $=$ Cocos nucifera, Cmil $=$ Cola millenii, Cafr $=$ Commiphora africana, Cada $=$ Crateva adansonii, Ccuj $=$ Crescentia cujete, Cgra $=$ Croton gratissimus, Dreg $=$ Delonix regia, Darb = Dracaena arborea, Egui $=$ Elaeis guineensis, Esen $=$ Erythrina senegalensis, Ecam = Eucalyptus camaldulensis, Etor = Eucalyptus torrelliana, Fexa = Ficus exasperata, Fing = Ficus ingens, Fumb = Ficus umbellata, Gter $=$ Gardenia ternifolia, Gsep = Gliricidia sepium, Garb = Gmelina arborea, Hflo = Holarrhena floribunda, Jmul = Jatropha multifida, Ksen = Khaya senegalensis, Lcup = Lecaniodiscus cupanioides, Mind = Mangifera indica, Mluc = Morinda lucida, Mole = Moringa oleifera, Nlae = Newbouldia laevis, Pbig = Parkia biglobosa, Pame = Persea americana, Pabi = Picea abies, Plon = Polyalthia longifolia, Pgua = Psidium guajava, Rbre = Rhodognaphalon brevicuspe Ssia $=$ Senna siamea, Smom = Spondias mombin, Tgra = Tectona grandis, Tvog = Tephrosia vogelii, Tcat $=$ Terminalia catappa, Tman $=$ Terminalia mantaly, Tsup = Terminalia superba, Tscl = Triplochiton scleroxylon, Vamy = Vachellia amythethophylla, Vdon = Vitex doniana.

According to the plant species ubiquity, the results showed that the RZ+RBZ shared the greatest number of species $(9$ plants) compared to the RZ+IZ (4 plants) and IZ+RBZ (3 plants), (Figure 3). Ubiquitous species groups contributed to $82.92 \%$ of all individuals recorded. The plant species of residential zone contributed the most $(55.18 \%)$ and the five largest being Mangifera indica, Citrus sinensis, Cocos nucifera, Ficus umbellata and Moringa oleifera. Of these species, only Ficus umbellata was native. The plant species of the road buffer zone contributed to $30.96 \%$ with a high presence of Cocos nucifera, Khaya senegalensis, Azadirachta indica and Terminalia catappa. The contribution of the ubiquitous plant species of the institutional zone was only $13.86 \%$.

By the plant species uniqueness, the residential zone had the highest number of unique plant species (17 plants) than the institutional zone (6 plants) and the road buffer zone (1 plant). The non-ubiquitous species groups contributed to $17.08 \%$ of all individuals. Of the 17 unique plant species recorded in the residential zone, 9 plants were native and 8 were exotic (Table 1). In the institutional zone, half of the 6 unique plant species were native (Triplochiton scleroxylon, Rhodognaphalon brevicuspe and Holarrhena floribunda) and the others were exotic (Polyalthia longifolia, Eucalyptus torrelliana and
Picea abies). Only Ceiba pentandra was unique to the road buffer zone (Table 1).

There was a significant link ( $\mathrm{p}$-value $=0.000)$ between the three strata (Table 4). This association was moderate intensity between residential zone and both road buffer and institutional zones, while it was quite strong between road buffer and institutional zones.

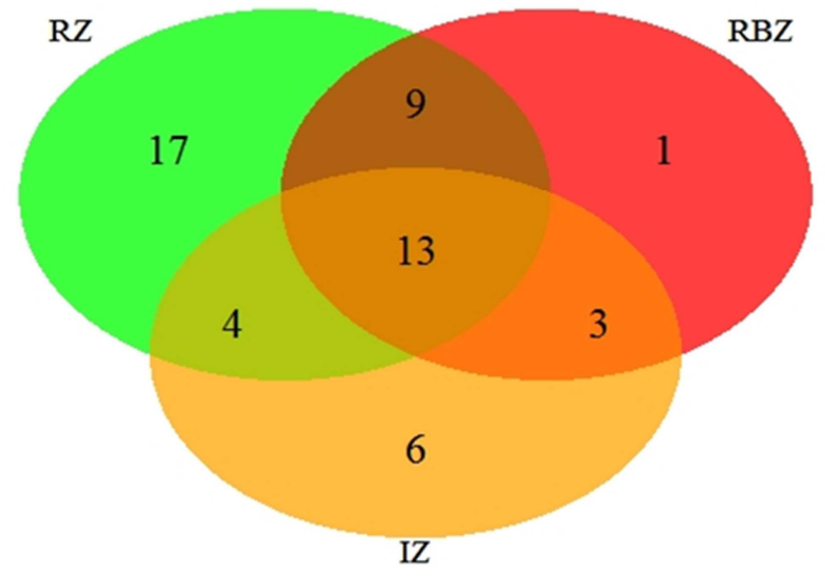

Figure 3. Venn diagram showing the number of species shared between urban strata (RZ-Residential zone: green, IZ-Institutional zone: orange, and RBZRoad buffer zone: red). 
Table 3. Frequency of plant species in the seven commonality groups.

\begin{tabular}{lllll}
\hline Species commonality groups & RBZ & IZ & RZ & Total \\
\hline RZ & 0 & 0 & 130 & 130 \\
$\mathrm{IZ}$ & 0 & 21 & 0 & 21 \\
$\mathrm{RBZ}$ & 2 & 0 & 0 & 2 \\
$\mathrm{RZ}+\mathrm{IZ}$ & 0 & 8 & 21 & 29 \\
$\mathrm{RZ}+\mathrm{RBZ}$ & 52 & 0 & 102 & 154 \\
$\mathrm{IZ}+\mathrm{RBZ}$ & 3 & 4 & 0 & 7 \\
$\mathrm{RZ}+\mathrm{IZ}+\mathrm{RBZ}$ & 175 & 91 & 287 & 553 \\
Total & 232 & 124 & 540 & 896 \\
\hline
\end{tabular}

Table 4. Cramer's V statistic from the Chisq association test, based on the frequency of plant species in Table 3.

\begin{tabular}{llll}
\hline Urban strata & RBZ & IZ & RZ \\
\hline RBZ & 1 & & \\
IZ & $0.41^{* * *}$ & 1 & \\
RZ & $0.35^{* * *}$ & $0.38^{* * *}$ & 1 \\
\hline
\end{tabular}

***: Significance level $<0.001$

\subsection{Plant Species Richness}

On the whole stands, 53 plants species were recorded. The species with an extremely low relative frequency were 21 plants and accounted for $39.46 \%$ of the total abundance (Table 1). Of the 53 plant species, 43 plants were identified in the residential zone while 26 were equivalently recorded in the institutional and road buffer zones.

The ten most predominant plant species were: Mangifera indica, Cocos nucifera, Citrus sinensis, Gliricidia sepium, Ficus umbellata, Azadirachta indica, Khaya senegalensis, Moringa oleifera, Ficus ingens and Terminalia catappa (Table 1). These species accounted for $69.31 \%$ of all individuals inventoried. The distribution of the preponderant plant species varied slightly between strata. Indeed, this category of plants accounted for $72.59 \%$ of all individuals in the residential zone (Gliricidia sepium, Mangifera indica, Citrus sinensis, Cocos nucifera, Ficus umbellata, Moringa oleifera, Ficus ingens, Azadirachta indica, Tectona grandis and Acacia auriculiformis). In the road buffer zone, they accounted for $81.47 \%$ of the total abundance (Cocos nucifera, Azadirachta indica, Khaya senegalensis, Terminalia catappa, Gmelina arborea, Ficus umbellata, Mangifera indica, Tectona grandis, Citrus sinensis and Elaeis guineensis). Finally, they contributed to $83.06 \%$ of all individuals in the institutional zone (Khaya senegalensis, Mangifera indica, Citrus sinensis, Terminalia catappa, Cocos nucifera, Azadirachta indica, Polyalthia longifolia, Triplochiton scleroxylon, Terminalia superba and Croton gratissimus).

\subsection{Plant Species Heterogeneity}

The profile of Shannon diversity index values showed that it was relatively high and fairly close to their maximum entropy value (Table 5). The three strata were relatively diversified. The $H^{\prime}$ value was lower with greater variation in the institutional zone than the other two strata. In addition, the analysis of variance performed on the $H^{\prime}$ value revealed a significant difference between residential zone and the both road buffer zone and institutional zones (Table 6). On the other hand, a non-significant difference was observed between road buffer and institutional zones.

Moreover, there was a strong regularity of individuals within species in the strata $\left(\mathrm{J}^{\prime}<0.80\right)$. This phenomenon was slightly more accentuated in the road buffer and institutional zones than in the residential zone.

The probability that two randomly selected individuals belonged to two different species was high within each stratum (IDS < 0.90). It means that 90 pairs out of 100 individuals taken at random were composed of different species. This trend was even more pronounced for the whole stands (Table 5).

Table 5. Indicators of species diversity in urban strata.

\begin{tabular}{llllll}
\hline Urban strata & $\mathbf{H}^{\prime}$ & $\boldsymbol{\sigma}\left(\mathbf{H}^{\prime}\right)$ & $\mathbf{H}_{\max }^{\prime}$ & $\mathbf{J}^{\prime}$ & SDI \\
\hline RBZ & 3.97 & 0.08 & 4.70 & 0.84 & 0.93 \\
IZ & 3.91 & 0.12 & 4.70 & 0.83 & 0.92 \\
RZ & 4.38 & 0.06 & 5.43 & 0.81 & 0.93 \\
All areas & 4.61 & 0.05 & 5.73 & 0.81 & 0.94 \\
\hline
\end{tabular}

Table 6. Significance of the difference on $H^{\prime}$ value between urban strata.

\begin{tabular}{lllll}
\hline Urban strata & RBZ & IZ & RZ & All areas \\
\hline RBZ & & ns & $* * *$ & $* * *$ \\
IZ & ns & & $* * *$ & $* * *$ \\
RZ & $* * *$ & $* * *$ & & $* * *$ \\
All areas & $* * *$ & $* * *$ & $* * *$ & \\
\hline
\end{tabular}

***: Significance level $<0.001$; ns: Not significant at 0.05 .

\subsection{Similarity Between Urban Strata}

The values of similarity indices in the whole stands were 0.646 and 0.780 for Jaccard and Sørensen indices, respectively. Regarding the values of Jaccard index between pair of strata, we noticed that they varied from $0.520 \pm 0.05$ (IZ-RZ) to $0.787 \pm 0.06$ (RBZ-IZ). As for the values of Sørensen index, they varied from $0.684 \pm 0.05$ (IZ-RZ) to $0.881 \pm 0.04$ (RBZIZ) (Figure 4). The similarity was stronger between the road buffer and institutional zones (similarity $\geq 0.7$ for Jaccard, and $\geq 0.8$ for Sørensen). It was moderate between the road buffer and residential zones, and relatively low between the institutional and residential zones.

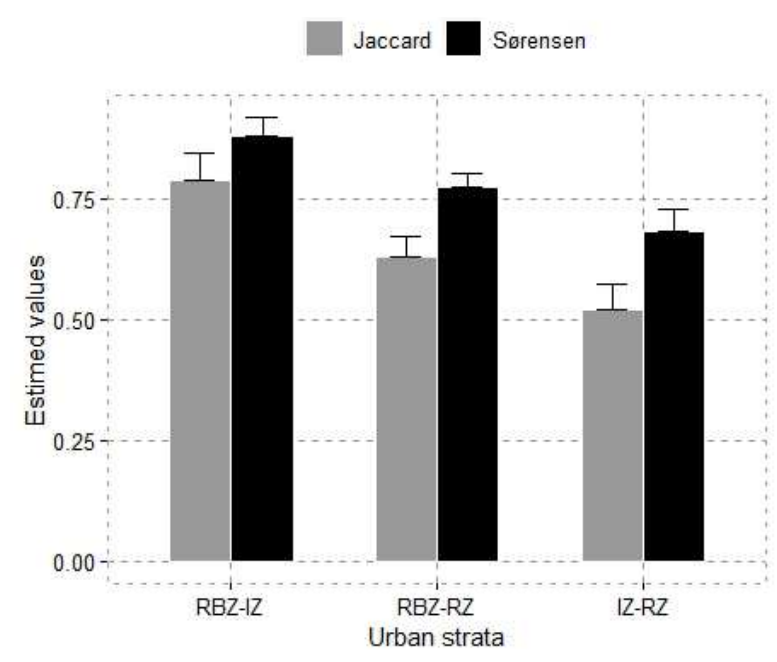

Figure 4. Compositional similarity between urban strata. 


\section{Discussion}

\subsection{Taxonomic Diversity of Plant Species}

The whole stands have 12.25 times more Dicotyledonous than Monocotyledonous. The high proportion of Dicotyledonous in urban flora had also been reported in previous works $[49,50]$. The taxonomic richness recorded in the strata belonged to the range of 20 to 52 families founded in the urban area of African regions [15, 21, 32, 49-53]. In this study, Leguminosae, Moraceae, Lamiaceae, Myrtaceae, Arecaceae, Combretaceae and Meliaceae were found to be the predominant families. Similar findings to ours were reported in the floristic composition of several cities, where they enjoyed the same status [15, 49-51, 54-57]. The number of families obtained in the road buffer zone is in the range of 14 to 27 families mentioned for others cities [30, 55-57]. That of the residential zone is lower than the 25 families reported for Kinshasa City [28]. The comparison of the present floristic list with those of previous works $[28,32,49-51,53,56]$ revealed that they charred 10 to 19 common families. This resemblance might be due to the high plasticity of the species of these families and the colonial history of the different cities. Moreover, the difference in the family rank-based on the number and importance of plant species-is relatively small between strata. The floristic traits of residential zone are similar to those of road buffer zone, whereas they differ significantly from those of institutional zone. The predominance of the same taxa in the three strata explained the observed correlation.

\subsection{Plant Species Importance}

The plant species importance value is relatively different between strata. For the whole stands, 20 plant species are a major ecological importance. Fourteen of them have to be considered as general preponderant plant species (IVI: 13 to $58 \%$ ) [58]. They are both abundant and frequent in the strata. The moderate and significant values obtained for the coefficient of correlation between the road buffer zone and the two other strata (institutional and residential zones), showed that these strata contained a few number of same predominant plant species (Khaya senegalensis, Mangifera indica, Cocos nucifera, Azadirachta indica, Citrus sinensis, Ficus umbellate, Terminalia catappa and Elaeis guineensis). Additionally, four of these generally preponderant plant species are native (Khaya senegalensis, Ficus umbellata, Ficus ingens and Elaeis guineensis). These species, with exception to Elaeis guineensis, did not come from relict forests in the fringe of urban area. They were savannah plant species that had been introduced into the city by some people and foresters. They benefited from environmental conditions generated by the Dahomean Gap phenomenon to establish themselves outside of their natural habitats. The importance of these plant species would be explained by their resistance for environmental stress, the attitude of population and foresters toward them and their management practices. It would be worthwhile to assess the potential for carbon sequestration and others regulating services of these plant species category.

\subsection{Plant Species Differentiation}

The native plant species are only higher of one unit than exotic ones (Exotic/Native: 49.06\%/50.94\%). However, exotic plant species are more abundant $(70.42 \%)$ and better distributed than native (29.58\%). Based on the origin, the urban flora of Lokossa is mixed with a relatively high introduction of exotic plant species. Thereby, these species contributed greatly to the plant species pool of the city $[8,19$, $59,60]$. The trend observed in the origin of plant species was in the same direction as that (Exotic/Native: 11\%/89\%) highlighted in Accra (Ghana) [61]. It was different from those mentioned in Lome City (Exotic/Native: 69\%/31\%) [50], Ziguinchor City (Exotic/Native: 53\%/47\%) [51] and Kinshasa City (Exotic/Native: $79.30 \% / 20.70 \%$ ) [28]. This difference of view was explained by the level of development, the multiculturalism and the morphology of Lokossa City. Lokossa is a medium-size city, fairly dense with more than ten ethnic groups [36]. It has large gaps that provide space for planting native and exotic plant species. According to [62], mixed character of the city flora contributed to the reduction of the air pollution. Contrariwise, a high proportion of exotic plant species could fundamentally alter the structure and function of the urban ecosystem $[63,64]$. It also influenced the conservation of native species and the disappearance of several animal species which depended on them [32]. The persistence of some plant species of pre-existing urban vegetation provided a relevant indicator of their sustainability in this urban area. Therefore, they should be studied and highlighted in planting projects of the city development plan.

With regard to stratum, the proportion of exotic plant species is $48.84 \%$ in the residential zone, $50 \%$ in the institutional zone and $53.85 \%$ in the road buffer zone. The predominance of exotic species in the road buffer zone was consistent with the results obtained in Kumasi Metropolis (Exotic/Native: 64.29\%/35.71\%) [56] and Bangalore (Exotic/Native: 67\%/33\%) [65]. The variation in the proportion of exotic plant species between strata is related to the management practices, planting decision-making process and the level of knowledge of the managers on the floristic composition of the urban area. Indeed, in the road buffer and institutional zones, plant species are selected by the forest administration service or site managers. They often tended to favor exotic plant species for amenity and aesthetics [21, 38]. In doing so, they contributed to a floristic homogeneity of these strata. On the other hand, the floristic composition of residential zone is influenced by attitude, cultural values and food needs of the populations $[59,66]$. Which favored the acceptance and the establishment of native plant species in the urban area [67]. The use of native plant species increased biological diversity by creating wildlife corridors. While strengthening the sense of belonging to nature [56]. Furthermore, these plant species were better adapted to local climatic conditions and lived in harmony with some harmful species (insects, parasites, fungi, etc.) [56]. Although, city dwellers tolerated the planting of native plant species by allowing them to grow on their home, they did not have 
enough information or knowledge to identify and recognize the functional traits, the growth dynamics and negative externalities they might generate $[15,67]$. These difficulties made populations and even political authorities to opt for exotic plant species.

\subsection{Plant Species Commonality and Uniqueness}

The high proportion (54.72\%) of ubiquitous plant species tend to uniform the urban forests physiognomy in the strata. The number of plant species shared by the residential and road buffer zones is higher than that shared by the institutional zone with the two others. The interaction of environmental conditions between strata and the choice of species planted in these sites would explain the distribution of ubiquitous plant. The floristic association of the residential zone with both road buffer and institutional zones is the result of a ripple effect caused by the attitude of the managers of the latter two strata. An afforestation attitude centered on the choice of ubiquitous species [38].

Otherwise, the strata have 3.85 to $39.53 \%$ unique plant species. They are made of several native and exotic plant species (12 specimens). Nevertheless, the frequency of these species is lower than that of ubiquitous species $(82.92 \%)$. The unique species helped to know the particularity of each stratum. Thereby, they give an indication on the choice of species and the mode of management of the strata. The residential zone has the largest cohort of unique species $(55.18 \%)$. The predominance of non-ubiquitous and native species in the residential zone is related to the persistent of plant species that were defended by cultural norms. The free choice of populations to plant species that were useful to them and the systematic protection of emblematic species were also worth mentioning.

\subsection{Plant Species Richness}

The plant species richness (53 plants) of the whole stands is relatively high. This value is in the range of 26 to 297 plant species found in several cities of Africa [15, 21, 26, 28, 32, 49-53, 64]. The ten most represented plant species accounted for $69.31 \%$ of all individuals. The forests stock of strata is made of a small number of dominant plant species including Mangifera indica, Cocos nucifera, Citrus sinensis, Gliricidia sepium, Ficus umbellata, Azadirachta indica, Khaya senegalensis, Moringa oleifera, Ficus ingens and Terminalia catappa. These results agreed with those of several authors $[15,21,25,26,32,49,51-53]$, who also highlighted the predominance of species such as Azadirachta indica, Mangifera indica, Cocos nucifera, Moringa oleifera, Khaya senegalensis, Terminalia spp, Citrus spp. The present plant species list shared 18 to 31 common species with those reported in previous works [28, 32, 49, 51, 53, 56].

The plant species richness of the strata is ranged from 26 to 43 plants. The residential zone is the richest (43 plants) compared to 26 plant species equivalently recorded in the road buffer and institutional zones. The species richness of residential zone is in the range of 29 to 70 species reported in the residential zone of several cities [15, 21, 26, 28, 32, 52]. Likewise, those of the road buffer zone is in the margin of 12 to 45 species recorded for streets forests $[15,21,26,30,32,52$, 55-57]. The institutional zone has low species richness compared to the range of 29 to 79 species obtained in the similar areas $[15,21,26,32,52]$. The ten predominant species in the three strata accounted for $72.59 \%$ to $83.06 \%$ of all individuals. This margin was higher than the value of $61 \%$ mentioned in Kumasi Metropolis [56]. Most of the species present in each of these strata were also mentioned as predominant in the streets forests $[15,21,26,50,52,56]$, the institutional zone $[15,21,26,52]$, and the residential zone [15, $21,26,28,50,52]$. The difference in species richness and abundance was related to the planting and management policy promoted by local authorities in each country.

\subsection{Plant Species Heterogeneity}

The plant species diversity observed in Lokossa City is high. Shannon's diversity index, evenness and Simpson's diversity index are greater than 4.5 bits, 0.80 and 0.90 , respectively. Therefore, the distribution of plant species abundance is not depended on one or two species in any given stratum. This high diversity also reflects the good health of the forests in the strata. Which presaged of the resilience of most plant species to different environmental disturbances and the regularity of urban ecosystem functions. The value of Shannon's diversity index obtained for the whole stands is higher than the range of 2.45 to 3.72 bits reported in others cities [21, 26, 32, 52, 53]. The value of the evenness is much higher than the value of 0.54 to 0.60 mentioned in Niamey and Maradi Cities [32] and Abuja City [53]. However, it is in the range of 0.72 to 0.954 recorded in Accra and Kumasi Cities [21, 26, 52]. The value of Simpson's diversity index is very close to those of 0.950 to 0.956 reported in previous works $[21,26,52]$. Although, all these studies were carried out in the cities with different climatic conditions, they were characterized by a higher species richness and a relatively low Shannon diversity index value compared to those obtained in this work. This would be explained by a high equality of individual contributions of the plant species in Lokossa City. The results on the diversity were consistent with the findings in African cities [60]. The importance of native plant species, the morphology of city, the level of development of city neighborhoods [59, 67], the population density [21,52] would also explained the plant species diversity of the strata. Maintaining this species diversity was imperative to improve vegetation cover, trees population tolerance to environmental diseases and stresses [11] and local microclimate [31]. The diversity was also necessary for minimizing the need of plant maintenance [11]. Education and public awareness of the benefits and ecosystem services of urban flora were important aspects of maintaining plant species diversity [53].

Moreover, it could be seen that the residential zone is globally more diversified than the road buffer and institutional zones. The difference in diversity between these strata is probably due to the choice of plant species and the forests management practices. The value of H' recorded in the residential zone is higher than the 
range of 2.49 to 3.56 bits' reported in the forests of several cities $[15,21,26,28,32,52,68]$. Its $\mathrm{J}$ ' value is in the range of 0.46 to 0.90 mentioned by these same authors. The value of IDS in the forests of these stratum was in the margin of 0.88 to 0.94 obtained in Accra and Kumasi Cities [21, 26, 52], and Kinshasa [28]. In the institutional zone, the value of $\mathrm{H}^{\prime}$ is higher than the margin of 2.67 to 3.84 bits reported in previous works $[15,21,26,28,32$, 52]. The value of $\mathrm{J}$ ' is in the range of 0.68 to 0.84 reported by the same previous authors. The value of IDS in the forests of these stratum was closed to the margin of 0.95 to 0.96 mentioned in Accra and Kumasi cities [21, 26, 52]. As for the values of H' obtained for the road buffer zone, it is in the range of 1.87 to 4.73 bits mentioned in several cities [1, 15, 21, 26, 30, 32, 52]. Likewise, the J' value of this stratum is in the margin of 0.53 to 0.89 resulting from the work of the same authors. The value of IDS in the road buffer zone is slightly higher than the margin of 0.829 to 0.903 reported in the cities of Accra and Kumasi [21, 26, 52].

The high diversity of residential zone would be explained by the attitudes and preferences of homeowners [59], socio-cultural factors $[69,70]$, socio-economic characteristics of households, level of education, freedom in choice of species $[32,50,68,71]$, species multi-functionality $[72,73]$, planting method [74], and socio-ecological constraints [21, 26, $52,75]$. It also revealed the importance of residential forests in the conserving biodiversity in the cities [59]. Additionally, the plant species diversity in the road buffer and institutional zones is very useful to avoid phytosanitary problems [76] in these strata.

\subsection{Similarity Between Urban Strata}

The values of the Jaccard (0.65) and Sørensen (0.78) similarity indices showed some dissimilarity between strata in Lokossa City. The pairwise combination of strata revealed a strong similarity (Jaccard $\geq 0.7$ and Sørensen $\geq 0.8$ ) between the road buffer zone (RBZ) and institutional zone (IZ). Structuring urban landscape on the basis of the compositional similarity revealed a decrease in similarity value when starting from the road buffer zone to residential zone (RBZ-IZ $>$ RBZ-RZ $>$ IZ-RZ). This similarity between strata would be explained by the high proportion of exotic ubiquitous plant species $(61.76 \%$ of ubiquitous plant species recorded). The attitude of sites managers or homeowners, the choice of species in planting projects and the forest management practices also explained the observed similarity. In the road buffer and institutional zones, managers were more concerned with aesthetics than the conservation of native species. Therefore, they chose exotic plant species, which leaded to a floristic homogeneity and a reduction of the dissimilarity. The similarity between the three strata was also mentioned for the urban area of Accra and Kumasi [21, 26].

\section{Implications for Plant Species Conservation Planning}

The context of climate changes and rapid urban growth require special attention to the flora in the city development plan, because the good condition of a city rests on the stability of its ecosystem [2]. One of the goals of city development plan would have to be the maintenance of this stability by species conservation planning. Such studies are useful for promoting the importance of flora in the cities. They can encourage local policy makers to have an ambitious development plan, which is no longer only devoted to the exploitation of natural resources and the collection of taxes. However, the consideration of the flora requires multidisciplinary knowledge (ecology, urban planning, arboriculture, local development, geomatics and database) and the cooperation between several professionals. Additionally, planting objectives should focus on improving the characteristics of the urban environment, enhancing the landscape, reducing air pollution and strengthening ecological functions of the urban ecosystem [1, 77]. To strengthen the species conservation planning in city development plan of Lokossa, the following points may be considered:

The policy makers and forests management offices have to invest sufficient resources for planting and monitoring projects of the flora. They must mandate professionals to draft planting and monitoring projects, to allocate clear and precise budget lines for each activity. They will establish a protocol for the implementation of the activities planned by the projects, which constituted an asset for improving the development plan in the context of global changes. Likewise, they will facilitate the allocation and capture funds from technical and financial partners to the urban development projects.

The choice of plant species and their monitoring will be made with reference to plausible scientific knowledge.

To diversify the urban flora, the decision-making process has to be focused on the native plant species. The flora of Benin has 30,700 plant species [78]. So, there are some potential candidates for planting projects.

The urban forests of Lokossa City have a large number of common species. To participate more actively in the conservation of native species with high potential, diversify the physiognomy of the forests and reduce the homogenization of flora, different trees identities can be assigned to city neighborhoods. At that scale, we can create an integral protected areas or participatory botanical gardens to act the adhesion of the population to best management practices.

The flora of road buffer zone is very homogeneous. This urban stratum is prone to several socio-environmental and spatial constraints. Therefore, the species choose for planting should be likely to: (1) adapt to the quality of the soil, (2) have medium sized crowns, (3) have a pivoting root dynamic and (4) be able to resist stress.

The species are better protected in the institutional zone. In this stratum, the native plant species must be easily promoted.

The residential zone is more diversifying. This stratum should be integrated into forest preservation and management initiatives in the city. Homeowners must be educated and encouraged to plant native species in their private gardens in 
order to increase global diversity and storage of biomass in the city.

The data collected in this work should be used as a test for creating a floristic database. This database will be regularly updated for a monitoring of urban flora. The information resulting from the monitoring will help to improve the urban forest management projects in the city development plan.

\section{Conclusions}

This study provided useful information on the urban flora of Lokossa. The composition and diversity of plants species differed relatively between strata. The whole stands have a high taxonomic diversity with predominance families of Leguminosae, Arecaceae, Malvaceae, Moraceae, Combretaceae, Lamiaceae and Myrtaceae. The urban flora is mixed $(50.94 \%$ of native species versus $49.06 \%$ of exotic species). Nevertheless, exotic species are more preponderant in the strata. The ubiquitous plant species represented $54.72 \%$ of the species pool and contributed to $82.92 \%$ of all individuals. The intensity of plant species association is quite high between road buffer and institutional zones. The flora of Lokossa City is very diversifying. The residential zone is more diversified than the road buffer and institutional zones. Furthermore, the road buffer and institutional zones are more similar (Jaccard $\geq 0.7$ and Sørensen $\geq 0.8$ ). The beta diversity of Lokossa City is relatively low.

Future research could evaluate the environmental benefits and services of plant species in Lokossa City. It would be worthwhile to study the impact of sprawling development on the natural forest relic of Lokossa in order to avoid their degradation and loss of valuable species.

\section{Acknowledgements}

The authors are grateful to the headmasters of the Scientific Council (CS), Laboratory of Applied Ecology (LEA) and Laboratory of Cartography (LaCarto) of Abomey-Calavi University (UAC) for the administrative, technical and financial support. Our thanks to the MOVIC team: Brice A. H. Tente, Ismaïla Toko Imorou, Ibouraïma Yabi, Waris K. Chouti, Mama Djaouga, David M. Baloubi and Emilia M. Azalou Tingbe for their availability and advice. We would like to thank Barthelemy C. Azankpe for his assistance with forest inventory. We also thank Aminou Ouorou and Marius Yetin for their assistance with language style and grammatical errors checking.

\section{References}

[1] Zhang, D., Zheng, H., He, X., Ren, Z. B., Zhai, C., Yu, X., Mao, Z. \& Wang, P. (2016). Effects of forest type and urbanization on species composition and diversity of urban forest in Changchun, Northeast China. Urban Ecosystems. 19, 455-473. doi: 10.1007/s11252-015-0473-5.

[2] Lv, H., Yang, Y., Zhang, D., Du, H., Zhang, J., Wang, W. \& He, X. (2019). Perimeter-area ratio effects of urbanization intensity on forest characteristics, landscape patterns and their associations in Harbin City, Northeast China. Urban Ecosystems. 22, 631-642. doi: 10.1007/s11252-019-00850-0.

[3] Langley, B. (2012). Modelling Urban Forest Structure and Services Using the Urban Forest Effects (UFORE) Model. Bachelor of science in forestry, University of British Columbia, Vancouver, Canada.

[4] Wang, W., Zhang, B., Xiao, L., Zhou, W., Wang, H.-F. \& He, X. (2018). Decoupling forest characteristics and background conditions to explain urban-rural variations of multiple microclimate regulation from urban trees. Peer J. 6, e5450. doi: 10.7717/peerj.5450.

[5] McKinney, M. L. (2002). Urbanization, Biodiversity, and Conservation: The impacts of urbanization on native species are poorly studied, but educating a highly urbanized human population about these impacts can greatly improve species conservation in all ecosystems. BioScience. 52 (10), 883-890.

[6] Conway, T. M. \& Bourne, K. S. (2013). A comparison of neighborhood characteristics related to canopy cover, stem density and species richness in an urban forest. Landsc. Urban Plan 113, 10-18. doi: 10.1016/j.landurbplan.2013.01.005.

[7] Kenney, W. A., van Wassenaer, P. J. \& Satel, A. L. (2011). Criteria and indicators for strategic urban forest planning and management. Arboriculture \& Urban Forestry. 37 (3), 108117.

[8] Nock, A. C., Paquette, A., Follett, M., Nowak, D. J. \& Messier, C. (2013). Effects of urbanization on tree species functional diversity in Eastern North America. Ecosystems. 16, 14871497. doi: 10.1007/s10021-013-9697-5.

[9] Alvey, A. A. (2006). Promoting and preserving biodiversity in the urban forest. Urban Forestry \& Urban Greening. 5 (4), 195-201. doi: 10.1016/j.ufug.2006.09.003.

[10] Kendal, D., Dobbs, C. \& Lohr, V. I. (2014). Global patterns of diversity in the urban forest: is there evidence to support the 10/20/30 rule? Urban Forestry \& Urban Greening. 13 (3), 411-417. doi: 10.1016/j.ufug.2014.04.004.

[11] Zainudin, S. R., Mustafa, K. A., Austin, D., Helmy, J. \& Lingkeu, D. A. (2012). Urban trees Diversity in Kuching North City and UNIMAS, Kota Samarahan, Sarawak. Pertanika Journal of Tropical Agricultural Science, 35 (1), 27-32.

[12] Shashua-Bar, L., Pearlmutter, D. \& Erell, E. (2011). The influence of trees and grass on outdoor thermal comfort in a hot-arid environment. International Journal of Climatology. 31 (10), 1498-1506. doi: 10.1002/joc.2177.

[13] Teka, O., Togbe, C. E., Djikpo, R., Chabi, R. \& Djossa, B. (2017). Effects of Urban Forestry on the Local Climate in Cotonou, Benin Republic. Agriculture, Forestry and Fisheries. Vol. 6, ํㅜ 4, 123-129. doi: 10.11648/j.aff.20170604.13.

[14] Ochola, E. M., Ochieng, A. A., Bosco, J. N., Mwibanda, J. W. \& Sahar, S. (2018). Attenuation effect of plant canopy sizes on microclimate in urban greenspaces within Nairobi City, Kenya. African Journal of Plant Science. 12 (7), 129-140. doi: 10.5897/AJPS2018.1659.

[15] Folega, F., Atakpama, W., Kanda, M., Konate, D., Gmadjom, K., Wala, K. \& Akpagana, K. (2019). Flora of urban green space of Atakpame city in Togo. Synthese: Revue des Science et de la Technologie. 25 (2), 25-39. 
[16] Ozkan, Y. U., Ozdemir, I., Saglam, S., Yesil, A. \& Demirel, T. (2016). Evaluating the Woody Species Diversity by Means of Remotely Sensed Spectral and Texture Measures in the Urban Forests. Journal of the Indian Society of Remote Sensing. 44, 687-697. doi: 10.1007/s12524-016-0550-0.

[17] United Nation (2016). Urbanization and development: emerging futures. United Nations: New York, USA.

[18] McDonnell, M. J., Pickett, S. T. A. \& Pouyat, R. V. (1993). The application of the ecological gradient paradigm to the study of urban effects. In Humans as components of ecosystems: subtle human effects and the ecology of populated areas. McDonnell, M. J., Pickett, S. T. A. (eds). Springer: New York, USA, pp. 175-189. doi: 10.1007/978-1-4612-0905-8 15.

[19] Walker, J. S., Grimm, N. B., Briggs, J. M., Gries, C. \& Dugan, L. (2009). Effects of urbanization on plant species diversity in central Arizona. Frontiers in Ecology and the Environment. 7 (9), 465-470. doi: 10.1890/080084.

[20] Williams, N. S. G., Schwartz, M. W., Vesk, P. A., McCarthy, M. A., Hahs, A. K., Clemants, S. E., Corlett, R. T., Duncan, R. P., Norton, B. A., Thompson, K. \& McDonnell, M. J. (2009). A conceptual framework for predicting the effects of urban environments on floras. Journal of Ecology. 97 (1), 4-9. doi: 10.1111/j.1365-2745.2008.01460.x.

[21] Nero, B. F., Nana Afranaa, K., Jatta, R. \& Fatunbi, O. (2018). Tree Species Diversity and Socioeconomic Perspectives of the Urban (Food) Forest of Accra, Ghana. Sustainability. 10 (3417), 1-19. doi: 10.3390/su10103417.

[22] Kühn, I. \& Klotz, S. (2006). Urbanization and homogenization: comparing the floras of urban and rural areas in Germany. Biological Conservation. 127 (3). 292-300. doi: 10.1016/j.biocon.2005.06.033.

[23] Ikin, K., Knight, E., Lindenmayer, D. B., Fischer, J. \& Manning, A. D. (2013). The influence of native versus exotic streetscape vegetation on the spatial distribution of birds in the suburbs and reserves. Diversity and Distributions. 19 (3), 294306; doi: 10.1111/j.1472-4642.2012.00937.x.

[24] Barth, B. J., Fitzgibbon, S. I. \& Wilson, R. S. (2015). New urban developments that retain more remnant trees have greater bird diversity. Landscape and Urban Planning. 136, 122-129. doi: 10.1016/j.landurbplan.2014.11.003.

[25] Fuwape, J. A. \& Onyekwelu, J. C. (2011). Urban Forest Development in West Africa: Benefits and Challenges. Journal of Biodiversity and Ecological Sciences. 1 (1), 77-94.

[26] Nero, B. F., Campion, B. B., Agbo, N., Callo-Concha, D. \& Denich, M. (2017). Tree and trait diversity, species coexistence, and diversity-functional relations of green spaces in Kumasi, Ghana. Procedia Engineering. 198, 99-115. doi: 10.1016/j.proeng.2017.07.164.

[27] Shackleton, C. M. (2012). Is there no urban forestry in the developing world? Scientific Research and Essays. 7 (40), 3329-3335. doi: 10.5897/SRE11.1117.

[28] Sambieni, K. R., Moyene, A. B., Toyi, M. S., Occhiuto, R., Bogaert, J. \& Dossou, B. (2018). La végétation arborée domestique dans le paysage urbain et périurbain de la ville de Kinshasa, République Démocratique du Congo. Afrique Science. 14 (2), 197-208.

[29] Amontcha, M. A. A., Lougbegnon, T., Tente, B., Djego, J. \& Sinsin, A. B. (2015). Aménagements urbains et dégradation de la phytodiversité dans la Commune d'Abomey-Calavi (Sud-Bénin). Journal of Applied Biosciences. 91, 8519-8528. doi: 10.4314/jab.v91i1.9.

[30] Osseni, A. A., Sinsin, A. B. \& Teka, O. (2014). Facteurs de contrôle de la diversité des plantations d'alignements dans la ville de Porto-Novo au Bénin. Afrique Science. 10 (4), 200-208.

[31] Teka, O. S., Togbe, C. E., Djossa, B. A., Djikpo, V. A. R., Oumorou, M. \& Sinsin, A. B. (2017). Plant diversity and carbon sequestration in roadside trees in Cotonou (Republic of Benin). Annales des Sciences Agronomiques. 21 (2), 203-221.

[32] Moussa, S., Kuyah, S., Kyereh, B., Tougiani, A. \& Mahamane, S. (2020). Diversity and structure of urban forests of Sahel cities in Niger. Urban Ecosystems. 23, 851-864. doi: 10.1007/s11252-020-00984-6.

[33] Arnberger, A., Brigitte, A., Eder, R., Ebenberger, M., Wanka, A., Kolland, F., Wallner, P. \& Hutter, H.-P. (2016). Elderly resident's uses of and preferences for urban green spaces during heat periods. Urban Forestry \& Urban Greening. 21, 102-115. doi: 10.1016/j.ufug.2016.11.012.

[34] Kabisch, N., Qureshi, S. \& Haase, D. (2015). Humanenvironment interactions in urban green spaces-a systematic review of contemporary issues and prospects for future research. Environmental Impact Assessment Review. 50, 25-34. doi: 10.1016/j.eiar.2014.08.007.

[35] Zhang, D., Wang, W. J., Zheng, H. F., Ren, Z. B., Zhai, C., Tang, Z., Shen, G. Q. \& He, X. Y. (2017). Effects of urbanization intensity on forest structural taxonomic attributes, landscape patterns and their associations in Changchun, Northeast China: implications for urban green infrastructure planning. Ecological Indicators. 80, 286-296. doi: 10.1016/j.ecolind.2017.05.042.

[36] National Institute of Statistic and Economic Analysis (2015). RGPH4: What to retain from the population in 2013? Service of Demographic studies, Cotonou, Benin.

[37] Nowak, D. J., Crane, E. D., Stevens, J. C., Hoehn, R. E., Walton, J. T. \& Bond, J. (2008). A Ground-Based Method of Assessing Urban Forest Structure and Ecosystem Services. Arboriculture \& Urban Forestry. 34 (6), 347-358.

[38] Jim, C. Y. (2002). Heterogeneity and differentiation of the tree flora in three major land uses in Guangzhou City, China. Annals of Forest Science. 59 (1), 107-118. doi: 10.1016/s0378-1127 (00)00449-7.

[39] Godefroid, S. \& Koedam, N. (2007). Urban plant species patterns are highly driven by density and function of built-up areas. Landscape Ecology. 22 (8), 1227-1239. doi: 10.1007/s10980-007-9102-x.

[40] Forthofer, R. N., Lee, E. S. \& Hernandez, M. (2007). Biostatistics: a guide to design, analysis and discovery. $2 \mathrm{nd}$ ed. Elsevier: USA, pp. 502.

[41] Curtis, J. T. \& Macintosh, R. P. (1951). An upland forest continuum in the prairie forest border region of Wisconsin. Ecology. 32 (3), 476-496. doi: 10.2307/1931725.

[42] Assogbadjo, A. E., Glèlè Kakaï, R. L., Sinsin, B. \& Pelz, D. (2009). Structure of Anogeissus leiocarpa Guill., Perr. natural stands in relation to anthropogenic pressure within Wari-Maro Forest Reserve in Benin. African Journal of Ecology. 48, 644653. doi: 10.1111/j.1365-2028.2009.01160.x. 
[43] Hammond, E. M. \& Pokorný, R. (2020). Diversity of Tree Species in Gap Regeneration under Tropical Moist Semi-Deciduous Forest: An Example from Bia Tano Forest Reserve. Diversity. 12 (301), 1-19. doi: 10.3390/d12080301.

[44] Magurran, A. E. (2004). Measuring ecological diversity. Blackwell publishing: Oxford, UK, pp. 256.

[45] Magurran, A. E. (1988). Ecological diversity and its measurement. 1st ed., Princeton University Press: New Jersey, USA, pp. 179.

[46] Chao, A., Chazdon, R. L., Colwell, R. K. \& Shen, T.-J. (2005). A new statistical approach for assessing similarity of species composition with incidence and abundance data. Ecology Lett. 8 (2), 148-159. doi: 10.1111/j.1461-0248.2004.00707.x.

[47] Chao, A., Chazdon, R. L., Colwell, R. K. \& Shen, T.-J. (2006). Abundance-based similarity indices and their estimation when there are unseen species in samples. Biometrics. 62 (2), 361371. doi: 10.1111/j.1541-0420.2005.00489.x

[48] R Core Team (2020). R: A language and environment for statistical computing; R Foundation for Statistical Computing, Vienna, Austria. URL https: //www.R-project.org/.

[49] N'Zala, D. \& Miankodila, P. (2002). Arbres et espaces verts à Brazzaville (Congo). Bois et Forest des Tropiques. 272 (2), 88 92.

[50] Radji, R., Kokou, K. \& Akpagana, K. (2011). Woody plant species used in urban forestry in West Africa: Case study in Lome, capital town of Togo. Journal of Horticulture and Forestry. 3 (1), 21-31. doi: 10.5897/JHF.9000120.

[51] Charahabil, M. M., Bassene, C., Balde, H., NDiaye, S. \& Diatta, M. (2018). Diversité et structure des espaces végétalisés urbains de la ville de Ziguinchor, Sénégal. International Journal of Biological and Chemical Sciences. 12 (4), 16501666. doi: 10.4314/ijbcs.v12i4.12.

[52] Nero, B. F., Callo-Concha, D. \& Denich, M. (2018). Structure, Diversity, and Carbon Stocks of the Tree Community of Kumasi, Ghana. Forests. 9 (519), 1-17. doi: 10.3390/19090519.

[53] Agbelade, A. D., Onyekwelu, J. C. \& Oyun, M. B. (2017). Tree Species Richness, Diversity, and Vegetation Index for Federal Capital Territory, Abuja, Nigeria. International Journal of Forestry Research. 2017, 1-12. doi: 10.1155/2017/4549756.

[54] Dardour, M., El Arbi, D., Azzouz, B., Nour-Eddine, K. \& Abdelbasset, B. (2014). Inventaire et état sanitaire des arbres d'alignement de la ville de Saïdia (Maroc oriental). Nature \& Technologie. 10, 2-9.

[55] Kouadio, Y. J. C., Vroh, B. T. A., Gone Bi, Z. B., Adou Yao, C. Y. \& N'Guessan, K. E. (2016). Evaluation de la diversité et estimation de la biomasse des arbres d'alignement des communes du Plateau et de Cocody (Abidjan-Côte d'Ivoire). Journal of Applied Biosciences. 97, 9141-9151. doi: 10.4314/jab.v97i1.1.

[56] Uka, U. N. \& Belford, E. J. D. (2016). Inventory of Street Tree Population and Diversity in the Kumasi Metropolis, Ghana. Journal of Forest and Environmental Sciences. 32 (4), 367 376. doi: 10.7747/JFES.2016.32.4.367.

[57] Kouassi, K. J., Kouassi, K. H. \& Kouassi, R. H. (2018). Evaluation de la diversité floristique et estimation du taux de séquestration de carbone des arbres en alignement de voies de la commune de Daloa (Côte d'Ivoire). International Journal of Biological and Chemical Sciences. 12 (4), 1876-1886. doi: 10.4314/ijbcs.v12i4.28.

[58] Mori, S. A., Boom, B. M., De Carvalino, A. M. \& Dos Santos, T. S. (1983). Southern Bahia moist forest. The Botanical Review. 49 (2), 155-232. doi: 10.1007/BF02861011.

[59] Nitoslawski, A. S. \& Duinker, N. P. (2016). Managing Tree Diversity: A Comparison of Suburban Development in Two Canadian Cities. Forests, 7 (119), 1-21. doi: 10.3390/f7060119.

[60] Murtala, D. \& Manaf, A. L. (2019). Progress and Methodological Approaches in Urban Trees and Forests Research in Africa. Journal of Environment and Earth Sciences. 9 (9), 32-45. doi: 10.7176/JEES/9-9-05.

[61] Simmering, D., Addai, S., Geller, G. \& Otte, A. (2013). A university campus in peri-urban Accra (Ghana) as a haven for dry-forest species. Flora Vegetatio Sudano-Sambesica. 16, 10 21. doi.org/10.21248/fvss.16.21.

[62] Nowak, D. J., Hirabayashi, S., Doyle, M., McGovern, M. \& Pasher, J. (2018). Air pollution removal by urban forests in Canada and its effect on air quality and Human Health. Urban Forestry \& Urban Greening. 29, 40-48. doi: 10.1016/j.ufug.2017.10.019.

[63] De Bruyn, O. (2016). Exotic plant species and environmental vulnerability: an old debate? The case study of Belgium (from the end of the 18th century to the 1950ies). VertigO. 16 (3), 122. https: //id.erudit.org/iderudit/1039984ar.

[64] Kimpouni, V., Nzila, J.-D., Watha-Ndoudy, N., Kokolo Bilongo, C. E., Yallo M. S., Kampe, J.-P. \& Louembe, D. (2020). Sociocultural and Ecological Dynamics of Green Spaces in Brazzaville (Congo). International Journal of Ecology. 2020,1-12. doi: 10.1155/2020/3719267.

[65] Nagendra, H. \& Gopal, D. (2010). Street trees in Bangalore: density, diversity, composition and distribution. Urban Forestry \& Urban Greening. 9 (2), 129-137. doi: 10.1016/j.ufug.2009.12.005.

[66] De Lacy, P. \& Shackleton, C. M. (2017). Aesthetic and spiritual ecosystem services provided by urban sacred sites. Sustainability. 9 (1628), 1-14. doi: 10.3390/su9091628.

[67] Doody, B. J., Sullivan, J. J., Meurk, C. D., Stewart, G. H. \& Perkins, H. C. (2010). Urban realities: The contribution of residential gardens to the conservation of urban forest remnants. Biodiversity and Conservation. 19 (5), 1385-1400. doi: 10.1007/s10531-009-9768-2.

[68] Wang, H.-F., Qureshi, S., Knapp, S., Friedman, C. R. \& Hubacek, K. (2015). A basic assessment of residential plant diversity and its ecosystem services and disservices in Beijing, China. Applied Geography. 64, 121-131. doi: 10.1016/j.apgeog.2015.08.006.

[69] Fraser, E. D. G. \& Kenney, W. A. (2000). Cultural background and landscape history as factors affecting perceptions of the urban forest. Journal of Arboriculture. 26 (2), 106-113.

[70] Almas, A. D. \& Conway, T. M. (2017). Residential Knowledge of Native Tree Species: A Case Study of Residents in Four Southern Ontario Municipalities. Environmental Management. 59 (1), 21-33. doi: 10.1007/s00267-016-0772-5. 
[71] Park, H., Kramer, M., Rhemtulla, J. M. \& Konijnendijk, C. C. (2019). Urban food systems that involve trees in northern America and Europe: a scoping review. Urban Forestry \& Urban Greening. 45, 126360. doi: 10.1016/j.ufug.2019.06.003.

[72] Galluzzi, G., Eyzaguirre, P. \& Negri, V. (2010). Home gardens: neglected hotspots of agro-biodiversity and cultural diversity. Biodiversity and Conservation. 19 (13), 3635-3654. doi: 10.1007/s10531-010-9919-5.

[73] Agbogidi, E. B. \& Adolor, O. M. (2014). Home gardens in the maintenance of biological diversity. Journal of Bio Innovation. $3(1), 04-19$.

[74] Nassauer, J. I., Wang, Z. \& Dayrell, E. (2009). What will the neighbors think? Cultural norms and ecological design. Landscape and Urban Planning. 92 (3-4), 282-292. doi: 10.1016/j.landurbplan.2009.05.010.
[75] Cilliers, S., Cilliers, J., Lubbe, R. \& Siebert, S. (2013). Ecosystem services of urban green spaces in African countriesperspectives and challenges. Urban Ecosystems. 16 (4), 681702. doi: 10.1007/s11252-012-0254-3.

[76] Bekkouch, I., Kouddane, N., Darouia, E., Boukroute, A. \& Berrichi, A. (2011). Inventaire des arbres d'alignement de la ville d'Oujda. Nature \& Technologie. 5, 87-91.

[77] Jim, C. Y. \& Liu, H. T. (2001). Species diversity of three major urban forest types in Guangzhou City, China. Forest Ecology and Management. 146 (1-3), 99-114. doi: 10.1016/s0378-1127 (00)00449-7.

[78] Akoègninou, A., van der Burg, W. J. \& van der Maesen, L. O. (2006). Flore Analytique du Benin. Backhuys Publishers: Wageningen, Pays-Bas, pp. 1035. 